\title{
Imunoexpressão da Proteina Her-2 em Punção Aspirativa com Ağuha Fina de Carcinoma de Mama: Correlação com os Achados da Peça Cirúrgica
}

\author{
Immunoexpression of Her-2 Protein in Fine-Needle Aspirates of Breast Carcinoma: \\ Correlation with Surgical Specimens
}

\author{
Marilene Almeida de Oliveira, Gilda da Cunha Santos, \\ Cristina Takami Kanamura, Venâncio Avancini Ferreira Alves.
}

\begin{abstract}
RESUM0
Objetivo: avaliar a imunoexpressão da proteína HER-2 em punção biópsia aspirativa de carcinoma de ductos infiltrantes da mama e nos espécimes cirúrgicos correspondentes. Correlacionar a imunoexpressão da proteína HER-2 com o grau histológico.

Método: foram examinados 48 esfregaços fixados em álcool, previamente corados pela técnica de Papanicolaou, e cortes histológicos de blocos de parafina do tecido correspondente. Todos foram submetidos à técnica de imuno-histoquímica, utilizando o método da avidina-biotinaperoxidase com um anticorpo policlonal (A0485-Dako). As reações foram realizadas simultaneamente com recuperação antigênica. A avaliação dos resultados foi feita por método semiquantitativo, segundo a graduação do HercepTest. Foram considerados positivos apenas os casos graduados em 3+.

Resultados: observou-se positividade de 39,6\% nos esfregaços e 35,4\% nos cortes histológicos respectivos, sendo a concordância cito-histológica nos casos positivos quase perfeita (kappa 0,82). A utilização do método de avaliação dos cortes histológicos mostrou-se reprodutivel nos esfregaços e padronizou a interpretação dos resultados. A correlação entre a positividade para proteína HER-2 e o grau histológico não foi significante, mas houve tendência a imunoexpressão mais freqüente em tumores menos diferenciados.

Conclusão: a positividade das reações imunocitoquímicas nos esfregaços foi comparável à obtida nos cortes histológicos, podendo portanto o material de punção aspirativa por agulha fina, previamente corado pela técnica de Papanicolaou, ser utilizado na avaliação da imunoexpressão da proteína HER-2.
\end{abstract}

PALAVRAS-CHAVE: Punção aspirativa por agulha fina. Oncogenes. Mama: carcinoma. Imunohistoquimica.

\section{Introdução}

O aprimoramento do conhecimento da biologia molecular tem permitido a obtenção de dados científicos mais pormenorizados sobre o com-

Departamento de Patologia Escola Paulista de Medicina UNIFESP (Universidade Federal de São Paulo) e Instituto Adolfo Lutz (São Paulo).

Correspondência:

Gilda da Cunha Santos

Departamento de Patologia Escola Paulista de Medicina Universidade Federal de São Paulo (UNIFESP)

Rua Botucatu, 740 - Vila Clementino

04023-900 - São Paulo - SP

e-mail: gildacs@patologia.epm.br portamento da célula tumoral e das etapas críticas do processo de desenvolvimento e progressão do câncer, com identificação de marcadores moleculares, acrescentando assim novos parâmetros utilizados como fatores prognósticos e preditivos do carcinoma de mama.

A procura por novos marcadores que possam ser preditivos é de importância crítica no desenvolvimento de terapias antineoplásicas. A família dos receptores de fator de crescimento epidérmico (c-erbB ou HER) é um dos grupos mais investigados atualmente.

O papel do HER-2 tem despertado grande interesse como indicador de resistência as várias formas de tratamento sistêmico para o câncer de 
mama. Há estudos associando a expressão do HER2 com resistência à quimioterapia citotóxica, como a ciclofosfamida/metrotrexato ${ }^{1}$. Outros relatos associam a expressão do HER-2 à melhor sensibilidade a outras drogas como a doxorrubicina ${ }^{2}$. Há também estudos relacionando a expressão do HER2 com resistência à terapia endócrina do câncer de mama com tamoxifeno ${ }^{1}$, devido à provável interação direta do HER-2 com receptores de estrógeno localizados na membrana plasmática ${ }^{3}$.

Em 1992, foi desenvolvido anticorpo monoclonal anti-HER-2 humanizado, que se liga com grande afinidade ao domínio extracelular do HER-2, inibindo a transmissão de sinais de estimulação ao crescimento ${ }^{4}$. Recentemente, este anticorpo monoclonal anti-HER-2 denominado trastuzumab (Herceptin ${ }^{\circledR}$, Genentech, San Francisco, CA) foi aprovado para uso comercial nos Estados Unidos e em países europeus ${ }^{5}$. Em estudos clínicos, o Herceptin, como agente terapêutico único, produziu resposta antitumoral objetiva em $15-20 \%$ das pacientes com metástase de carcinoma de mama, incluindo alguns casos com quimioterapia prévia ${ }^{6}$. Quando associado com outros quimioterápicos (doxorrubicina, ciclofosfamida ou paclitaxel), o Herceptin aumentou a eficácia da quimioterapia, com aumento geral da sobrevida ${ }^{7}$.

A terapêutica com Herceptin é efetiva apenas contra os tumores que mostram expressão aumentada do receptor HER-2 na superfície celular, sendo importante a identificação de pacientes que possam se beneficiar desta modalidade terapêutica, o que tem gerado interesse nas técnicas de identificação do receptor HER-2 no carcinoma de mama.

A punção aspirativa com agulha fina (PAAF) é método simples e, devido à elevada precisão diagnóstica, tem sido muitas vezes usado para indicação direta do tratamento. Desta forma, nas pacientes candidatas à terapia sistêmica prévia à ressecção cirúrgica, muitas vezes a amostra tumoral obtida por PAAF é a única fonte para pesquisa de marcadores biológicos de possivel eficácia terapêutica.

Tem-se demonstrado que a imunoexpressão da proteína HER-2 em esfregaços previamente corados pela técnica de Papanicolaou é comparável à obtida em cortes histológicos ${ }^{8-10}$.

Os objetivos do presente trabalho foram: avaliar a expressão imunocitoquímica da proteína HER-2 em esfregaços de PAAF de carcinoma ductal infiltrante da mama e sua correlação com os resultados nos espécimes cirúrgicos correspondentes, e comparar a imunoexpressão da proteína HER-2 com o grau histológico dos tumores.

\section{Pacientes e Métodos}

Foi realizado estudo retrospectivo no qual foram incluídos 48 casos de PAAF de mama feminina, de pacientes atendidas consecutivamente, com diagnóstico prévio positivo para carcinoma, no período de 1999 a 2001. As lâminas e os blocos de parafina foram obtidos do arquivo do Laboratório de Patologia Cirúrgica e Citopatologia Ltda (Governador Valadares, MG). Os casos foram analisados e selecionados como adequados para este trabalho de acordo com os seguintes critérios: diagnóstico citológico de carcinoma, confirmado posteriormente no exame histopatológico; esfregaços citológicos representativos fixados em álcool etílico 95\% e corados pela técnica de Papanicolaou; preparados histológicos e blocos de parafina de biópsia ou ressecção cirúrgica correspondente ao local da PAAF; fixação adequada do material histológico, e diagnóstico histopatológico de carcinoma ductal invasivo, confirmados após revisão.

Todos os preparados histológicos foram corados pela técnica de hematoxilina-eosina e analisados por um mesmo observador (MAO) em microscópio óptico comum. A graduação histológica foi baseada no sistema de Scarff-Bloom-Richardson modificado por Elston e Ellis ${ }^{11}$.

Os esfregaços e os cortes histológicos foram submetidos à técnica de imunohistoquímica utilizando o método avidina-biotina-peroxidase $(\mathrm{ABC})^{12}$, com recuperação antigênica utilizandose incubação em tampão citrato $10 \mathrm{mM}$, pH 6,0, em panela de pressão, por três minutos, sob pressão, a seguir deixando esfriar por 20 minutos em temperatura ambiente. A incubação com anticorpo primário biotinilado policlonal anti-HER A0485 (Dako, Dinamarca), na diluição 1:1000, foi realizada por 16 horas, durante a noite.

Os controles positivo (um caso de carcinoma mamário com imunoexpressão intermediária $(2+/ 3+)$ para HER-2) e negativo (um caso com omissão da etapa de incubação do anticorpo primário) foram realizados para todas as reações.

A interpretação da expressão dos marcadores foi feita de forma equivalente nas amostras citológicas e histológicas. Para avaliar a imunoexpressão da proteína HER-2, somente a intensidade e o padrão de coloração da membrana plasmática foram considerados, usando a mesma escala de avaliação semiquantitativa padronizada para o HercepTest (Dako), aprovado pela FDA, para terapêutica com o Herceptin ${ }^{5}$. As colorações citoplasmáticas foram consideradas não específicas.

Para avaliar a concordância entre os esfregaços e os cortes histológicos foram considerados positivos para a imunoexpressão da proteí- 
na HER-2 apenas os casos graduados em 3+ (fortemente positivos), já que estudos recentes demonstraram maior correlação da imunoexpressão da proteína HER-2, com amplificação do gene pela técnica de hibridização in situ, nos casos graduados em $33^{+13-15}$. Utilizou-se a medida de kappa ${ }^{16}$, que é baseada no número de respostas concordantes, ou seja, o mesmo nos dois métodos. O kappa corresponde à medida de concordância, onde o valor 0 indica nenhuma concordância e o valor 1 representa total concordância. Foi usado como parâmetro para $o \kappa$ estatístico a graduação relativa dividida por Landis e Koch ${ }^{17}$. Para medir a relação entre a expressão do HER-2 e o grau histológico foi aplicado o teste do $\chi^{2}$ Pearson e, quando conveniente, o teste exato de Fisher. Foi adotado o nivel de significância de 0,05 (5\%).

O projeto de pesquisa deste estudo foi avaliado e aprovado pelo Comitê de Ética em Pesquisa da Universidade Federal de São Paulo.

\section{Resultados}

Das 48 pacientes incluídas neste estudo, 16 eram pré-menopausadas e 32 menopausadas, com média de idade de 54 anos.

Em relação à graduação histológica, 7 dos carcinomas ductais infiltrantes foram considerados grau I (14,5\%), 23 (48\%) grau II e $18(37,5 \%)$ grau III.

$\mathrm{Na}$ avaliação dos esfregaços, observou-se a seguinte distribuição: 19 casos $(39,6 \%)$ graduados em $3+, 17$ casos $(35,4 \%)$ em $2+, 4(8,3 \%)$ em $1+$ e 8 $(16,7 \%)$ em 0.

A intensidade da coloração foi difusa e homogênea nos casos positivos (3+) para a expressão da proteína HER-2, sendo observada em alguns esfregaços uma menor intensidade da reação nas células da periferia da lâmina em relação às centrais. Em dois esfregaços hemorrágicos e com obscurecimento celular parcial, a interpretação dos resultados foi possível em células isoladas na periferia dos blocos celulares.

A avaliação histológica revelou 10 casos $(20,8 \%)$ classificados como 0,9 casos $(18,8 \%)$ como $1+, 12$ casos $(25 \%)$ como $2+$ e 17 casos $(35,4 \%)$ como $3+$.

Houve variação da intensidade da reação em dois casos nos quais o componente in situ, tipo comedo, foi graduado em 3+ e a área de invasão em $2+$, sendo esta última considerada como a representativa do caso.

O valor de kappa na avaliação citohistológica quando comparados os resultados em geral foi $\kappa=0,595$ (Tabela 1) e quando considerados como positivos apenas os casos $3+$, o valor de kappa mostrou-se bastante significativo, $\kappa=0,823$ (Tabela 2).

Tabela 1 - Concordância entre a expressão da proteína HER-2 nos esfregaços e nos cortes histológicos correspondentes.

\begin{tabular}{lllcccc}
\hline & & \multicolumn{5}{c}{ Citologia } \\
& & $\mathbf{0}$ & $\mathbf{1 +}$ & $\mathbf{2 +}$ & $\mathbf{3 +}$ & Total \\
\hline Histologia & 0 & 6 & 2 & 2 & & 10 \\
& $1+$ & 2 & 2 & 4 & 1 & 9 \\
& $2+$ & & & 10 & 2 & \\
& $3+$ & & & 1 & 16 & \\
Total & & 8 & 4 & 17 & 19 & 48 \\
\hline
\end{tabular}

kappa $=0,595$

Tabela 2 - Concordância cito-histológica categorizada em duas classes: negativo $(0,1+, 2+)$ e positivo $(3+)$ para expressão da proteína HER-2.

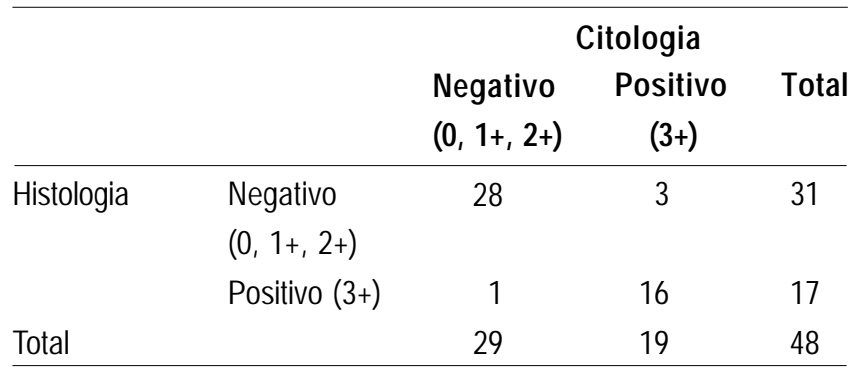

kappa $=0,823$

Não foi observada correlação significativa entre a imunoexpressão do HER-2 e o grau histológico, na peça cirúrgica (Tabela 3). À medida que o grau histológico aumentou, observou-se uma maior quantidade de casos positivos, porém esse resultado não foi estatisticamente significante $(p=0,1740)$. Vale ressaltar que nenhum dos carcinomas bem diferenciados (grau I) mostrouse positivo com intensidade de $3+$.

\section{Discussão}

O câncer de mama é resultado de alterações moleculares que são geneticamente e/ou externamente induzidas, resultando em proliferação celular desordenada. Uma das alterações moleculares significativas observadas é a amplificação do gene HER-2 e conseqüente super-expressão da proteína HER-2 ${ }^{18}$. Neste estudo, avaliou-se a imunoexpressão da proteína HER-2 em esfregaços 
Tabela 3 - Correlação da expressão da proteína HER-2 na peça cirúrgica com o grau histológico.

\begin{tabular}{|c|c|c|c|c|c|c|c|c|c|c|}
\hline \multicolumn{11}{|c|}{ Histologia HER-2 } \\
\hline & & \multicolumn{2}{|c|}{0} & \multicolumn{2}{|c|}{$1+$} & \multicolumn{2}{|c|}{$2+$} & \multicolumn{2}{|c|}{$3+$} & \multirow[t]{2}{*}{ Tota } \\
\hline & & $\mathrm{n}$ & $\%$ & $\mathrm{n}$ & $\%$ & $\mathrm{n}$ & $\%$ & $\mathrm{n}$ & $\%$ & \\
\hline Grau & 1 & 4 & 51,7 & 2 & 28,6 & 1 & 14,3 & 9 & 39,1 & 7 \\
\hline \multirow[t]{2}{*}{ Histológico } & II & 4 & 17,4 & 4 & 17,4 & 6 & 26,1 & 8 & 44,4 & 23 \\
\hline & III & 2 & 11,1 & 3 & 16,7 & 5 & 27,8 & 17 & & 18 \\
\hline Total & & 10 & & 9 & & 12 & & & & 48 \\
\hline
\end{tabular}

$p=0,1740 \quad$ Teste exato de Fisher

fixados em álcool e previamente corados pela técnica de Papanicolaou. Os resultados mostraram concordância quase perfeita entre a positividade do HER-2 nos cortes histológicos e nos esfregaços correspondentes, com discordância em três casos. Resultados semelhantes foram observados em um trabalho no qual se estudaram 50 esfregaços fixados em álcool 95\% e previamente corados pela técnica de Papanicolaou, com índices de positividade de $34 \%$ na citologia e $32 \%$ na histologia ${ }^{8}$. Outro trabalho, com 47 amostras similares, obteve correlação completa com índice de positividade de $21 \%^{10}$. Ambos verificaram, nos casos positivos, padrão uniforme de coloração em todas as células neoplásicas, concordante com o presente estudo, o que torna este fenômeno de particular interesse em citologia aspirativa da mama, principalmente em amostras paucicelulares.

O emprego de diferentes técnicas de fixação e diferentes anticorpos tem levado a ampla variação de resultados. Positividade de $28 \%$ na citologia e $20 \%$ na histologia correspondente foi descrita utilizando-se esfregaços fixados em Carnoy ${ }^{9}$. Com citocentrifugado de material obtido por PAAF em suspensão celular em PBS, fixado em acetona e congelado, obtiveram-se índices de positividade de $48 \%$ na citologia e $43 \%$ na histologia ${ }^{19}$. Em esfregaços de punção, secos e congelados, observaram-se $37,9 \%$ de positividade na citologia e $16,7 \%$ na histologia correspondente ${ }^{20}$. A comparação entre raspados de espécimes cirúrgicos fixados em álcool 95\%, esfregaços secos e os cortes em parafina mostrou 73,67 e $47 \%$ de positividade, respectivamente ${ }^{21}$.

Usando a técnica de FISH (hibridização in situ fluorescente) como padrão-ouro, outros autores compararam esfregaços de raspados de espécimes cirúrgicos fixados em álcool 95\% e os cortes histológicos correspondentes. Obtiveram índices de sensibilidade, especificidade e probabilidade de positividade de $88,8,77,7$ e $80 \%$ para a histologia e 69, 87 e 90\% para citologia, respectivamente, com concordância de $77 \%$ entre citologia e histologia ${ }^{22}$.
A maior positividade em esfregaços citológicos do presente estudo, como em outros trabalhos, tem sido relacionada à fixação em álcool, situação em que há melhor preservação antigênica que no tecido fixado em formol e incluído em parafina ${ }^{8,9,21,22}$. Os preparados citológicos também têm mostrado maior especificidade e sensibilidade, em comparação com a técnica de FISH, do que os cortes histológicos ${ }^{22}$, e os sinais de fluorescência podem ser melhor visibilizados e graduados nos esfregaços citológicos ${ }^{22,23}$.

A avaliação dos resultados do presente trabalho como em outros estudos, foi semiquantitativa. A maioria observou a positividade ou não da membrana e a intensidade da reação, se forte ou fraca $^{8-10}$. Outros avaliaram a positividade de acordo com a quantidade (porcentagem) de células co$\operatorname{radas}^{19,20,22}$.

As amostras citológicas do presente estudo foram submetidas às reações de imunocitoquímica, utilizando-se o mesmo anticorpo, recuperação antigênica e reações simultâneas com as amostras histológicas correspondentes. Foi escolhido o anticorpo policlonal A0485 (Dako), o mesmo utilizado no HercepTest. Utilizaram-se os mesmos critérios de avaliação dos resultados nos cortes histológicos e esfregaços, baseados no sistema de graduação do HercepTest, e considerando positivos apenas os casos $3+$. Trabalhos recentes demonstram que esta positividade imunohistoquímica 3+ apresenta elevada correlação com a amplificação do gene HER-2 pela técnica de FISH $^{15,23,24}$. A avaliação da imunoexpressão do HER2, utilizando diferentes anticorpos e a graduação do HercepTest, revelou alta amplificação do gene nos casos fortemente positivos (3+), independentemente do anticorpo ${ }^{14}$. Foi observada, em outro trabalho, correlação dos casos 3+ com tumores de grau histológico III, com baixos índices de positividade do estrógeno e piora geral do prognóstico ${ }^{25}$. Os casos fortemente positivos também têm sido associados a melhores respostas terapêuticas ao Herceptin ${ }^{15}$.

Atualmente, tem sido proposta a técnica de 
imuno-histoquímica para a seleção de casos completamente negativos ou fortemente positivos e a complementação com a técnica de FISH nos casos graduados em $2+{ }^{15,25}$. Há relatos de amplificação gênica em casos negativos e a falta de amplificação em casos fortemente positivos ${ }^{13}$. Portanto, o uso da técnica de FISH apenas nos casos graduados em 2+ não eliminaria todos os falso-positivos e falso-negativos.

Embora as correlações entre a positividade para o HER-2 e o grau de diferenciação histológica não tenham sido significativas nesse estudo, observou-se tendência da expressão do HER-2 nos tumores menos diferenciados (grau II e III), não sendo encontrado nenhum caso positivo em tumores bem diferenciados (grau I), o que já havia sido descrito por outros autores ${ }^{15}$. Significativa associação entre casos 3+ e tumores grau III tem sido relatada ${ }^{15,25}$.

Em conclusão, como a positividade imunocitoquímica em esfregaços mostrou alta correlação com os achados nos cortes histológicos, a PAAF poderá ser cada vez mais usada como fonte primária de material na avaliação do HER-2, especialmente em pacientes candidatas a quimioterapia adjuvante. Abre-se, também, a perspectiva de uso de amostras de PAAF na avaliação de novos fatores prognósticos e preditivos em neoplasias mamárias.

\section{ABSTRACT}

Purpose: to determine the expression of HER-2 protein in fine-needle aspirates obtained from infiltrating breast duct carcinoma and in the corresponding surgical specimens and to correlate HER-2 expression with histological grade. Methods: forty-eight smears fixed in alcohol and previously stained by the Papanicolaou technique and histological sections of corresponding paraffin-embedded tissue blocks were submitted to immunohistochemistry by the avidin-biotinperoxidase method using a polyclonal antibody (A0485Dako). The reactions were carried out simultaneously with antigen recovery. The results were analyzed by a semiquantitative method according to the HercepTest score. Scores of 3+ were considered to be positive.

Results: positivity was observed in $39.6 \%$ of the smears and in $35.4 \%$ of the respective histological sections, with almost perfect cytohistologic concordance (kappa 0.82). The method of evaluation of histological sections was found to be reproducible regarding the smears and standardized the interpretation of the results. The correlation between HER-2 positivity and histological grade was not statistically significant but there was a tendency towards HER-2 expression in less differentiated tumors.

Conclusion: the positivity of the immunocytochemical reactions observed in smears was comparable to that obtained in the histological sections, indicating that the material obtained by fine-needle aspiration biopsies previously stained by the Papanicolaou technique can be used for the evaluation of HER-2 expression.

KEY WORDS: Fine-needle aspiration cytology. Oncogene: HER-2 protein. Invasive ductal carcinoma. Immunohistochemistry.

\section{Referências}

1. Têtu B, Brisson J, Plante V, Bernard P. p53 and cerbB-2 as markers of resistance to adjuvant chemotherapy in breast cancer. Mod Pathol 1998; 11:823-30.

2. Paik S, Bryant J, Park C, et al. C-erbB-2 and response to doxorubicin in patients with axillary lymph nodepositive, hormone receptor-negative breast cancer. J Natl Cancer Inst 1998; 90:1361-70.

3. Chung YL, Sheu ML, Yang SC, Lin CH, Yen SH. Resistance to tamoxifen-induced apoptosis is associated with direct interaction between HER-2/ neu and cell membrane estrogen receptor in breast cancer. Int J Cancer 2002; 97:306-12.

4. Carter P, Presta L, Gorman CM, et al. Humanization of an anti-p185 HER-2 antibody for human cancer therapy. Proc Natl Acad Sci USA 1992; 89:4285-9.

5. Grazziano C. HER-2 breast assay, linked to herceptin wins FDA's okay. CAP Today 1998; 12:13-6.

6. Cobleigh MA, Vogel CL, Tripathy D, et al. Multinational study of the efficacy and safety of humanized anti-HER2 monoclonal antibody in women who have HER2-overexpressing metastatic breast cancer that has progressed after chemotherapy for metastatic disease. J Clin Oncol 1999; 17:2639-48.

7. Slamon D J, Leyland-Jones B, Shak S, et al. Use of chemotherapy plus a monoclonal antibody against HER-2 for metastatic breast cancer that overexpresses HER-2. N Engl J Med 2001; 344:783-92.

8. Jorda M, Ganjei P, Nadji M. Retrospective c-erbB-2 immunostaining in aspiration cytology of breast cancer. Diagn Cytopathol 1994; 11:262-5.

9. Corkill ME, Katz R. Immunocytochemical staining of C-erbB-2 oncogene in fine-needle aspirates of breast carcinoma: a comparison with tissue sections and other breast cancer prognostic factors. Diagn Cytopathol 1994; 11:250-4.

10.Martin AW, Davey DD. Comparison of immunoreactivity of neu oncoprotein in fine-needle aspirates and paraffin-embedded materials. Diagn Cytopathol 1995; 12:142-5. 
11.Elston CW, Ellis IO. Pathological prognostic factors in breast cancer. I. The value of histological grade in breast cancer. Histopathology 1991; 19:403-10.

12.Hsu SM, Raine L, Fanger H. Use of avidin-biotinperoxidase complex $(\mathrm{ABC})$ in immunoperoxidase technique: a comparison between $\mathrm{ABC}$ and unlabeled antibody (PAP) procedures. J Histochem Cytochem 1981; 29:577-80.

13.Pauletti G, Dandekar S, Rong H, et al. Assessment of methods for tissue-based detection of the HER$2 /$ neu alteration in human breast cancer: a direct comparison of fluorescence in situ hybridization and immunohistochemistry. J Clin Oncol 2000; 18:3651-64.

14.Lebeau A, Deimling D, Kaltz C, et al. HER-2/neu analysis in archival tissue samples of human breast cancer: comparison of immunohistochemistry and fluorescence in situ hybridization. J Clin Oncol 2001; 19:354-63.

15. Field AS, Chamberlain NL, Tran D, Morey AL. Suggestions for HER-2/neu testing in breast carcinoma, based on a comparison of immunohistochemistry and fluorescence in situ hybridization. Pathology 2001; 33:278-82.

16.Agresti A. Categorical data analysis. $1^{\text {st }}$ ed. New York: Wiley; 1990.

17.Landis JR, Koch GG. The measurement of observer agreement for categorical data. Biometrics 1977; 33:159-74.

18.Slamon DJ, Clark GM, Wong SG, Levin WJ, Ullrich A, McGuire WL. Human breast cancer: correlation of relapse and survival with amplification of the HER-2/neu oncogene. Science 1987; 235:177-82.
19.Troncone G, Panico L, Vetrani A, et al. C-erbB-2 expression in FNAB smears and matched surgical specimens of breast cancer. Diagn Cytopathol 1996; 14:135-9.

20.Tiniakos DG, Robinson KB, Greenwood H, et al. CerbB-2 and p53 expression in breast cancer fine needle aspirates. Cytopathology 1996; 7:178-86.

21.Solomides CC, Zimmerman R, Bibbo $M$. Semiquantitative assessment of c-erbB-2 (HER-2) status in cytology specimens and tissue sections from breast carcinoma. Anal Quant Cytol Histol 1999; 21:121-5.

22.McKee GT, Tambouret RH, Finkelstein D. A realiable method of demonstrating HER-2/neu estrogen receptors, and progesterone receptors on routinely processed cytologic material. Appl Immunohistochem Mol Morphol 2001; 9:352-7.

23.Jacobs TW, Gown AM, Yazigi H, Barnes MJ, Schnitt SJ. Comparison of fluorencence in situ hybridization and immunohistochemistry for the evaluation of HER-2/neu in breast cancer. J Clin Oncol 1999; 17:1974-82.

24.Rhodes A, Jasani B, Couturier J, et al. A formalinfixed, paraffin-processed cell line standard for quality control of immunohistochemical assay of HER-2/neu expression in breast cancer. Am J Clin Pathol 2002; 117:81-9

25.Birner P, Oberhuber G, Stani J, et al. Evaluation of the United States Food and Drug Administrationapproved scoring and test system of HER-2 protein expression in breast cancer. Clin Cancer Res 2001; 7:1669-75.

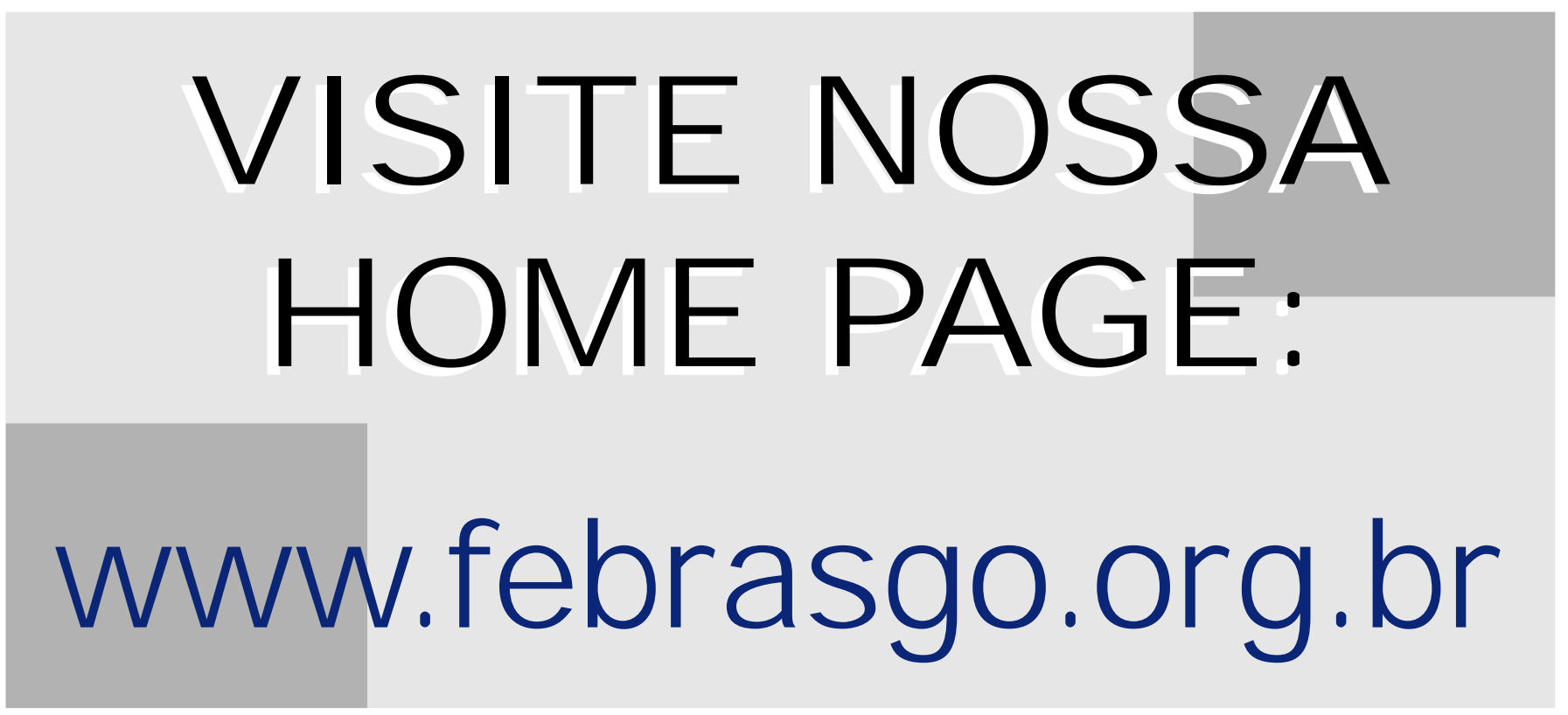

